CTP/TAMU-33/92

\title{
A Multimonopole Solution in String Theory ${ }^{\dagger}$
}

\author{
Ramzi R. Khuri* \\ Center for Theoretical Physics \\ Texas A\&M University \\ College Station, TX 77843
}

\begin{abstract}
A multimonopole solution in Yang-Mills field theory is obtained by a modification of the 't Hooft ansatz for a four-dimensional instanton. Although this solution has divergent action near each source, it can be used to construct an exact finite action multimonopole solution of heterotic string theory, in which the divergences from the Yang-Mills sector are precisely cancelled by those from the gravity sector.
\end{abstract}

$4 / 92$

$\dagger$ Work supported in part by NSF grant PHY-9106593.

* Supported by a World Laboratory Fellowship. 


\section{Introduction}

In recent work [1] [], several classical solitonic solutions of string theory with instanton structure have been presented. Exact solutions in bosonic [1,2] and heterotic [6, [7] string theory have been obtained by observing that a generalized curvature 8,90 combining the metric and antisymmetric tensor possesses instanton structure[1].

In this paper we present an exact multimonopole solution of heterotic string theory. We first obtain an analogous mutlimonopole solution in Yang-Mills field theory, whose action diverges near each source. We show that the string theory solution, however, is finite as a result of the cancellation of divergent terms from the gauge and gravitational sectors.

In section 2 we derive a multimonopole solution of four-dimensional YM + scalar field theory via a modification of the 't Hooft ansatz 10 14] for the Yang-Mills instanton. This solution is not in the Prasad-Sommerfield [15] limit, but has the topology of $Q=1$ monopole sources, saturates the Bogomoln'yi bound[16] and exhibits the far field behaviour of multimonopole sources. The action for this solution, however, diverges near each source.

We use this solution in section 3 to construct an exact heterotic multimonopole solution. This string solution has finite action, as the divergences coming from the Yang-Mills sector are precisely cancelled by those from the gravitational sector. The resultant action reduces to the tree-level form and is easily calculated.

A more detailed version of this letter is presented in [17].

\section{Multimonopole Solution in Field Theory}

Consider the four-dimensional Euclidean action

$$
S=-\frac{1}{2 g^{2}} \int d^{4} x \operatorname{Tr} G_{\mu \nu} G^{\mu \nu}, \quad \quad \mu, \nu=1,2,3,4 .
$$

For gauge group $S U(2)$, the fields may be written as $A_{\mu}=(g / 2 i) \sigma^{a} A_{\mu}^{a}$ and $G_{\mu \nu}=$ $(g / 2 i) \sigma^{a} G_{\mu \nu}^{a} \quad$ (where $\sigma^{a}, a=1,2,3$ are the $2 \times 2$ Pauli matrices). The equation of motion derived from this action is solved by the 't Hooft ansatz[10 14]

$$
A_{\mu}=i \bar{\Sigma}_{\mu \nu} \partial_{\nu} \ln f
$$

where $\bar{\Sigma}_{\mu \nu}=\bar{\eta}^{i \mu \nu}\left(\sigma^{i} / 2\right)$ for $i=1,2,3$, where

$$
\begin{array}{rlrl}
\bar{\eta}^{i \mu \nu}=-\bar{\eta}^{i \nu \mu} & =\epsilon^{i \mu \nu}, & \mu, \nu=1,2,3, \\
& =-\delta^{i \mu}, & \nu & =4
\end{array}
$$


and where $f^{-1} \square f=0$. The ansatz for the anti-self-dual solution is similar, with the $\delta$-term in (2.3) changing sign.

To obtain a multi-instanton solution, one solves for $f$ in the four-dimensional space to obtain

$$
f=1+\sum_{i=1}^{N} \frac{\rho_{i}^{2}}{\left|\vec{x}-\vec{a}_{i}\right|^{2}},
$$

where $\rho_{i}^{2}$ is the instanton scale size and $\vec{a}_{i}$ the location in four-space of the $i$ th instanton.

We obtain a multimonopole solution by modifying this procedure as follows. We single out a direction in the transverse four-space ( say $x_{4}$ ) and assume all fields are independent of this coordinate. Then the solution for $f$ can be written as

$$
f=1+\sum_{i=1}^{N} \frac{m_{i}}{\left|\vec{x}-\vec{a}_{i}\right|},
$$

where $m_{i}$ is the charge and $\vec{a}_{i}$ the location in the three-space (123) of the $i$ th monopole. If we make the identification $\Phi \equiv A_{4}$ (we loosely refer to this field as a Higgs field in this paper, even though there is no apparent symmetry breaking mechanism), then the Lagrangian density for the above ansatz can be rewritten as

$$
\begin{aligned}
G_{\mu \nu}^{a} G_{\mu \nu}^{a} & =G_{i j}^{a} G_{i j}^{a}+2 G_{k 4}^{a} G_{k 4}^{a} \\
& =G_{i j}^{a} G_{i j}^{a}+2 D_{k} \Phi^{a} D_{k} \Phi^{a}
\end{aligned}
$$

which has the same form as the Lagrangian density for YM + massless scalar field in three dimensions.

We now go to $3+1$ dimensions with the Lagrangian density (signature $(-+++)$ )

$$
\mathcal{L}=-\frac{1}{4} G_{\mu \nu}^{a} G^{\mu \nu a}-\frac{1}{2} D_{\mu} \Phi^{a} D^{\mu} \Phi^{a}
$$

and show that the above multimonopole ansatz is a static solution with $A_{0}^{a}=0$ and all time derivatives vanish. The equations of motion in this limit are given by

$$
\begin{aligned}
D_{i} G^{j i a} & =g \epsilon^{a b c}\left(D^{j} \Phi^{b}\right) \Phi^{c}, \\
D_{i} D^{i} \Phi^{a} & =0 .
\end{aligned}
$$

It is then straightforward to verify that the above equations are solved by the modified 't Hooft ansatz

$$
\begin{aligned}
\Phi^{a} & =\mp \frac{1}{g} \delta^{a i} \partial_{i} \omega, \\
A_{k}^{a} & =\epsilon^{a k j} \partial_{j} \omega
\end{aligned}
$$


where $\omega \equiv \ln f$. This solution represents a multimonopole configuration with sources at $\vec{a}_{i}=1,2 \ldots N$. A simple observation of far field and near field behaviour shows that this solution does not arise in the Prasad-Sommerfield [15] limit. In particular, the fields are singular near the sources and vanish as $r \rightarrow \infty$. This solution can be thought of as a multi-line source instanton solution, each monopole being interpreted as an "instanton string" [18].

The topological charge of each source is easily computed $\left(\hat{\Phi}^{a} \equiv \Phi^{a} /|\Phi|\right)$ to be

$$
Q=\int d^{3} x k_{0}=\frac{1}{8 \pi} \int d^{3} x \epsilon_{i j k} \epsilon^{a b c} \partial_{i} \hat{\Phi}^{a} \partial_{j} \hat{\Phi}^{b} \partial_{k} \hat{\Phi}^{c}=1
$$

The magnetic charge of each source is then given by $m_{i}=Q / g=1 / g$. It is also straightforward to show that the Bogomoln'yi[16] bound

$$
G_{i j}^{a}=\epsilon_{i j k} D_{k} \Phi^{a}
$$

is saturated by this solution. Finally, it is easy to show that the magnetic field $B_{i}=$ $\frac{1}{2} \epsilon_{i j k} F^{j k}$ (where $F_{\mu \nu} \equiv \hat{\Phi}^{a} G_{\mu \nu}^{a}-(1 / g) \epsilon^{a b c} \hat{\Phi}^{a} D_{\mu} \hat{\Phi}^{b} D_{\nu} \hat{\Phi}^{c}$ is the gauge-invariant electromagnetic field tensor defined by 't Hooft[19]) has the the far field limit behaviour of a multimonopole configuration:

$$
B(\vec{x}) \rightarrow \sum_{i=1}^{N} \frac{m_{i}\left(\vec{x}-\vec{a}_{i}\right)}{\left|\vec{x}-\vec{a}_{i}\right|^{3}}, \quad \text { as } \quad r \rightarrow \infty
$$

As usual, the existence of this static multimonopole solution owes to the cancellation of the gauge and Higgs forces of exchange-the "zero-force" condition.

We have presented all the monopole properties of this solution. Unfortunately, this solution as it stands has divergent action near each source, and this singularity cannot be simply removed by a unitary gauge transformation. This can be seen for a single source by noting that as $r \rightarrow 0, A_{k} \rightarrow \frac{1}{2}\left(U^{-1} \partial_{k} U\right)$, where $U$ is a unitary $2 \times 2$ matrix. The expression in parentheses represents a pure gauge, and there is no way to get around the $1 / 2$ factor in attempting to "gauge away" the singularity 20. The field theory solution is therefore not very interesting physically. As we shall see in the next section, however, we can obtain an analogous finite action solution in string theory. 


\section{Exact Heterotic Multimonopole Solution}

We use the above solution to construct an exact multimonopole solution of heterotic string theory. The derivation of this solution closely parallels that of the multi-instanton solution presented in [6.7], but in this case, the solution possesses three-dimensional (rather than four-dimensional) spherical symmetry near each source. Again the reduction is effected by singling out a direction in the transverse space.

The tree-level supersymmetric vacuum equations for the heterotic string are given by

$$
\begin{gathered}
\delta \psi_{M}=\left(\nabla_{M}-\frac{1}{4} H_{M A B} \Gamma^{A B}\right) \epsilon=0, \\
\delta \lambda=\left(\Gamma^{A} \partial_{A} \phi-\frac{1}{6} H_{A M C} \Gamma^{A B C}\right) \epsilon=0, \\
\delta \chi=F_{A B} \Gamma^{A B} \epsilon=0,
\end{gathered}
$$

where $\psi_{M}, \lambda$ and $\chi$ are the gravitino, dilatino and gaugino fields. The Bianchi identity is given by

$$
d H=\alpha^{\prime}\left(\operatorname{tr} R \wedge R-\frac{1}{30} \operatorname{Tr} F \wedge F\right) .
$$

The $(9+1)$-dimensional Majorana-Weyl fermions decompose down to chiral spinors according to $S O(9,1) \supset S O(5,1) \otimes S O(4)$ for the $M^{9,1} \rightarrow M^{5,1} \times M^{4}$ decomposition. Let $\mu, \nu, \lambda, \sigma=1,2,3,4$ and $a, b=0,5,6,7,8,9$. Then the ansatz

$$
\begin{aligned}
g_{\mu \nu} & =e^{2 \phi} \delta_{\mu \nu}, \\
g_{a b} & =\eta_{a b}, \\
H_{\mu \nu \lambda} & = \pm \epsilon_{\mu \nu \lambda \sigma} \partial^{\sigma} \phi
\end{aligned}
$$

with constant chiral spinors $\epsilon_{ \pm}$solves the supersymmetry equations with zero background fermi fields provided the YM gauge field satisfies the instanton (anti)self-duality condition

$$
F_{\mu \nu}= \pm \frac{1}{2} \epsilon_{\mu \nu}^{\lambda \sigma} F_{\lambda \sigma}
$$

An exact solution is obtained as follows. Define a generalized connection by

$$
\Omega_{ \pm M}^{A B}=\omega_{M}^{A B} \pm H_{M}^{A B}
$$


embedded in an $\mathrm{SU}(2)$ subgroup of the gauge group, and equate it to the gauge connection $A_{\mu}$ [21] so that $d H=0$ and the corresponding curvature $R\left(\Omega_{ \pm}\right)$cancels against the YangMills field strength $F$. The crucial point is that for $e^{-2 \phi} \square e^{2 \phi}=0$ with the above ansatz, the curvature of the generalized connection can be written in the covariant form [1]

$$
\begin{aligned}
R\left(\Omega_{ \pm}\right)_{\mu \nu}^{m n}= & \delta_{n \nu} \nabla_{m} \nabla_{\mu} \phi-\delta_{n \mu} \nabla_{m} \nabla_{\nu} \phi+\delta_{m \mu} \nabla_{n} \nabla_{\nu} \phi-\delta_{m \nu} \nabla_{n} \nabla_{\mu} \phi \\
& \pm \epsilon_{\mu m n \alpha} \nabla_{\alpha} \nabla_{\nu} \phi \mp \epsilon_{\nu m n \alpha} \nabla_{\alpha} \nabla_{\mu} \phi
\end{aligned}
$$

from which it easily follows that

$$
R\left(\Omega_{ \pm}\right)_{\mu \nu}^{m n}=\mp \frac{1}{2} \epsilon_{\mu \nu}^{\lambda \sigma} R\left(\Omega_{ \pm}\right)_{\lambda \sigma}^{m n}
$$

Thus we have a solution with the ansatz (3.5) such that

$$
F_{\mu \nu}^{m n}=R\left(\Omega_{ \pm}\right)_{\mu \nu}^{m n}
$$

where both $F$ and $R$ are (anti)self-dual. This solution becomes exact since $A_{\mu}=\Omega_{ \pm \mu}$ implies that all the higher order corrections vanish 21,22,23]. The self-dual solution for the gauge connection is then given by the 't Hooft ansatz

$$
A_{\mu}=i \bar{\Sigma}_{\mu \nu} \partial_{\nu} \ln f
$$

A multimonopole is obtained as in the previous section by

$$
f=e^{-2 \phi_{0}} e^{2 \phi}=1+\sum_{i=1}^{N} \frac{m_{i}}{\left|\vec{x}-\vec{a}_{i}\right|}
$$

where $m_{i}$ is the charge and $\vec{a}_{i}$ the location in the three-space (123) of the $i$ th monopole. If we again identify the Higgs field as $\Phi \equiv A_{4}$, then the gauge and Higgs fields may be simply written in terms of the dilaton as

$$
\begin{aligned}
\Phi^{a} & =-\frac{2}{g} \delta^{i a} \partial_{i} \phi, \\
A_{k}^{a} & =-\frac{2}{g} \epsilon^{a k j} \partial_{j} \phi
\end{aligned}
$$

for the self-dual solution. For the anti-self-dual solution, the Higgs field simply changes sign. Here $g$ is the YM coupling constant. Note that $\phi_{0}$ drops out in (3.13).

The above solution (with the gravitational fields obtained directly from (3.5) and (3.12)) represents an exact multimonopole solution of heterotic string theory and has the 
same structure in the four-dimensional transverse space as the multimonopole solution of the YM + scalar field action of section 2. If we identify the (123) subspace of the transverse space as the space part of the four-dimensional spacetime (with some toroidal compactification, similar to that used in [24]) and take the timelike direction as the usual $X^{0}$, then the monopole properties described in the previous section carry over directly into the string solution.

The string action contains a term $-\alpha^{\prime} F^{2}$ which also diverges as in the field theory solution. However, this divergence is precisely cancelled by the term $\alpha^{\prime} R^{2}\left(\Omega_{ \pm}\right)$in the $O\left(\alpha^{\prime}\right)$ action. This result follows from the exactness condition $A_{\mu}=\Omega_{ \pm \mu}$ which leads to $d H=0$ and the vanishing of all higher order corrections in $\alpha^{\prime}$. Another way of seeing this is to consider the higher order corrections to the bosonic action shown in [22,23]. All such terms contain the tensor $T_{M N P Q}$, a generalized curvature incorporating both $R\left(\Omega_{ \pm}\right)$and $F$. The ansatz is contructed precisely so that this tensor vanishes identically [1,2]. The action thus reduces to its lowest order form and can be calculated directly for a multi-source solution from the expressions for the massless fields in the gravity sector.

The divergences in the gravitational sector in heterotic string theory thus serve to cancel the divergences stemming from the field theory solution. This solution thus provides an interesting example of how this type of cancellation can occur in string theory, and supports the promise of string theory as a finite theory of quantum gravity. Another point of interest is that the string solution represents a supersymmetric multimonopole solution coupled to gravity, whose zero-force condition in the gravity sector (cancellation of the attractive gravitational force and repulsive antisymmetric field force) arises as a direct result of the zero-force condition in the gauge sector (cancellation of gauge and Higgs forces of exchange) once the gauge connection and generalized connection are identified.

We now calculate the mass of the heterotic multimonopole configuration. Naively, the mass can be calculated from the tree-level action

$$
S=-\frac{1}{2 \kappa^{2}} \int d^{3} x \sqrt{g} e^{-2 \phi}\left(R+4(\nabla \phi)^{2}-\frac{H^{2}}{12}\right) .
$$

There is one subtlety we must consider, however (see [25]). From the term $\sqrt{g} e^{-2 \phi} R$ in the integrand of the action, the action density in (3.14) contains double derivative terms of the metric component fields. In general, one would like to work with an action which depends only on the fields and their first derivatives. This problem was solved in general relativity by Gibbons and Hawking [26,27], who added a surface term which precisely cancelled the 
double derivative terms in the action in general relativity. The addition of a surface term does not, of course, affect the equations of motion.

It turns out that there is a relatively straightforward generalization of the GibbonsHawking surface term (GHST) to string theory[28,29]. By antisymmetry, the axion field does not contribute to the GHST and the surface term in this case can be written in the simple form

$$
S_{G H S T}=-\frac{1}{\kappa^{2}} \int_{\partial M}\left(e^{-2 \phi} K-K_{0}\right)
$$

where $\partial M$ is the surface boundary and $K$ and $K_{0}$ are the traces of the fundamental form of the boundary surface embedded in the metric $g$ and the Minkowskian metric $\eta$ respectively. The correct effective action is thus obtained by adding the surface term of (3.15) to the volume term of (3.14):

$$
S=-\frac{1}{2 \kappa^{2}}\left[\int d^{3} x \sqrt{g} e^{-2 \phi}\left(R+4(\nabla \phi)^{2}-\frac{H^{2}}{12}\right)+2 \int_{\partial M}\left(e^{-2 \phi} K-K_{0}\right)\right] .
$$

By using the equations of motion, the volume term $S_{V}$ can be written as a surface term (see [25]):

$$
S_{V}=-\frac{1}{\kappa^{2}} \int_{\partial M} \hat{n} \cdot \vec{\nabla} e^{-2 \phi}
$$

Note that $\sqrt{g}$ has been absorbed into the surface measure of $\partial M$. Since we have separability of sources in the limit of surfaces of infinite radius, we may therefore compute $S_{V}$ for a single monopole configuration in three-space

$$
\begin{aligned}
e^{2 \phi} & =1+\frac{m}{r}, \\
g_{i j} & =e^{2 \phi} \delta_{i j},
\end{aligned}
$$

and simply add the contributions of an arbitrary number of sources. The contribution of a single monopole to the static volume action is given by

$$
\begin{aligned}
S_{V} & =-\frac{1}{\kappa^{2}}\left(\frac{\partial}{\partial r} e^{-2 \phi}\right) A(M) \\
& =-\frac{4 \pi m}{\kappa^{2}}
\end{aligned}
$$

in the $r \rightarrow \infty$ limit, where $A(M)=4 \pi r^{2}(1+m / r)$ is the area of the boundary surface.

We now turn to the GHST. A simple calculation of the extrinsic curvature $K$ for a single monopole configuration (3.18) gives

$$
K=\frac{2}{r^{2}} e^{-3 \phi}(r+m / 2)
$$


When the surface $\partial M$ is embedded in flat space, the radius of curvature $R$ is given by $R=r e^{\phi}$. The extrinsic curvature $K_{0}$ is then given by

$$
K_{0}=\frac{2}{R}=\frac{2}{r} e^{-\phi} .
$$

The GHST is therefore given by

$$
S_{G H S T}=-\frac{2}{\kappa^{2} r}\left(e^{-5 \phi}\left(1+\frac{m}{2 r}\right)-e^{-\phi}\right) A(M)=\frac{12 \pi m}{\kappa^{2}}
$$

in the $r \rightarrow \infty$ limit.

The total static action for a multi-soliton configuration, equal to the total mass of the solitons, can then be obtained by adding the static contributions to the action of the volume part and the GHST. The result is

$$
M_{T}=\frac{8 \pi}{\kappa^{2}} \sum_{n=1}^{N} m_{n}
$$

For our multimonopole configuration, however, it should be noted that $m_{n}=1 / \mathrm{g}$ for $n=1,2 \ldots N$.

\section{Conclusion}

In this paper, we presented an exact multimonopole solution of heterotic string theory. This solution represents a supersymmetric extension of the bosonic string multimonopole solution outlined in [30], and is obtained by a modification of the 't Hooft ansatz for a four-dimensional instanton. Exactness was shown by the generalized curvature method used in [1,2, 6, 7] to obtain exact instanton solutions in bosonic and heterotic string theory. Unlike the instanton solutions, however, the monopole solutions do not seem to be easily describable in terms of conformal field theories, an unfortunate state of affairs from the point of view of string theory.

An analogous multimonopole solution of the four dimensional field theory of YM + massless scalar field was first written down. This solution possesses the properties of a multimonopole solution (topology, far-field limit and Bogomoln'yi bound) but has divergent action near each source. We used this solution to construct the string theory solution, for which the divergences in the YM sector are cancelled by similar divergences in the gravity sector, resulting in a finite action solution. This finding is significant in that it represents an example of how string theory incorporates gravity in such a way as to cancel infinities inherent in gauge theories, thus supporting its promise as a finite theory of quantum gravity. 


\section{References}

[1] R. R. Khuri, Phys. Lett. B259 (1991) 261.

[2] R. R. Khuri, Some Instanton Solutions in String Theory to appear in Proceedings of the XXth International Conference on Differential Geometric Methods in Theoretical Physics, World Scientific, October 1991.

[3] M. J. Duff and J. X. Lu, Nucl. Phys. B354 (1991) 141.

[4] M. J. Duff and J. X. Lu, Nucl. Phys. B354 (1991) 129.

[5] A. Strominger, Nucl. Phys. B343 (1990) 167.

[6] C. G. Callan, J. A. Harvey and A. Strominger, Nucl. Phys. B359 (1991) 611.

[7] C. G. Callan, J. A. Harvey and A. Strominger, Nucl. Phys. B367 (1991) 60.

[8] R. R. Metsaev and A. A. Tseytlin, Phys. Lett. B191 (1987) 354.

[9] R. R. Metsaev and A. A. Tseytlin, Nucl. Phys. B293 (1987) 385.

[10] G. 't Hooft, Phys. Rev. Lett., 37 (1976) 8.

[11] F. Wilczek, in Quark confinement and field theory, Ed. D. Stump and D. Weingarten, John Wiley and Sons, New York (1977).

[12] E. Corrigan and D. B. Fairlie, Phys. Lett. B67 (1977) 69.

[13] R. Jackiw, C. Nohl and C. Rebbi, Phys. Rev. D15 (1977) 1642.

[14] R. Jackiw, C. Nohl and C. Rebbi, in Particles and Fields, Ed. David Boal and A. N. Kamal, Plenum Publishing Co., New York (1978), p.199.

[15] M. K. Prasad and C. M. Sommerfield, Phys. Rev. Lett. 35 (1975) 760.

[16] E. B. Bogomolnyi, Sov. J. Nucl. Phys. 24 (1976) 449.

[17] R. R. Khuri A Heterotic Multimonopole Solution, Texas A\&M preprint, CTP/TAMU$35 / 92$.

[18] P. Rossi, Physics Reports, 86(6) 317-362.

[19] G. 't Hooft, Nucl. Phys. B79 (1974) 276.

[20] R. Rajaraman, Solitons and Instantons, North Holland, 1982.

[21] M. Dine, Lectures delivered at TASI 1988, Brown University (1988) 653.

[22] E. A. Bergshoeff and M. de Roo, Nucl. Phys. B328 (1989) 439.

[23] E. A. Bergshoeff and M. de Roo, Phys. Lett. B218 (1989) 210.

[24] J. A. Harvey and J. Liu, Phys. Lett. B268 (1991) 40.

[25] R. R. Khuri, Manton Scattering of String Solitons PUPT-1270 (to appear in Nucl. Phys. B).

[26] G. W. Gibbons and S. W. Hawking, Phys. Rev. D15 (1977) 2752.

[27] G. W. Gibbons, S. W. Hawking and M. J. Perry, Nucl. Phys. B318 (1978) 141.

[28] S. B. Giddings and A. Strominger, Nucl. Phys. B306 (1988) 890.

[29] D. Brill and G. T. Horowitz, Phys. Lett. B262 (1991) 437.

[30] R. R. Khuri, Solitons and Instantons in String Theory, Princeton University Doctoral Thesis, August 1991. 